\title{
Laparoscopic Radical Cystectomy: a 5-year review of a single institute's operative data and complications and a systematic review of the literature
}

\author{
Omar M. Aboumarzouk, Tomasz Drewa, Pawel Olejniczak, Piotr L. Chlosta
}

Urology Department, Royal Bournemouth Hospital (OMA), Castle Lane East, Bournemouth, BH7 7DW, United Kingdom; Department of Urology, Institute of Oncology, Kielce and Department of Urology, Nicolaus Copernicus Hospital, Torun (TD, PO), Poland; Department of Urology, Institute of Oncology (PLC) UJK University, Kielce, Poland and Department of Urology, the Medical Centre of Postgraduate Education (PLC), Warsaw, Poland

\section{ABSTRACT}

Objective: We aim to evaluate our experience and results with laparoscopic radical cystectomy and conduct a systematic review of studies reporting on 50 or more procedures. Materials and Methods: Between February 2006 and March 2011, a prospective study in a single institute on patients with bladder cancer who underwent laparoscopic radical cystectomy was conducted.

A search of the Cochrane Library, PubMed, Medline, and Scopus databases was conducted for studies reporting on 50 or more laparoscopic radical cystectomy procedures to compare with our results.

Results: Sixty men and five women underwent laparoscopic radical cystectomy during the 5-year study period. Thirty-nine patients were submitted to ileal conduits, 24 to neobladders, and two patients to ureterocutaneostomies. The mean operative time was $294 \pm 27$ minutes, the mean blood loss was $249.69 \pm 95.59$ millilitres, the mean length of hospital stay was $9.42 \pm 2$ days, the mean morphine requirement was $3.69 \pm 0.8$ days.

The overall complication rate was $44.6 \%$ (29/65). However, the majority of the patients with complications (90\% (26/29)) had minor complications treated conservatively with no further surgical intervention needed.

The literature search found seven studies, which reported on their institutions' laparoscopic radical cystectomy results of 50 or more patients. Generally, our results were similar to other reported studies of the same calibre.

Conclusion: Laparoscopic radical cystectomy is a safe and efficient modality of treatment of bladder cancer. However, it comes with a steep learning curve, once overcome, can provide an alternative to open radical cystectomy.

\section{ARTICLE INFO}

\section{Key words:}

Laparoscopy; cystectomy;

lymph node dissection

Int Braz J Urol. 2012; 38: 330-40

Submitted for publication:

February 09, 2012

Accepted after revision:

March 30, 2012

\section{INTRODUCTION}

The gold standard for treatment of localized muscle-invasive, bladder cancer is open radical cystectomy (ORC) $(1,2)$. However, in the turn of the century, numerous centres have adopted a more minimally invasive approach (1-3). In 1993, de Badajoz et al. performed the first laparoscopic radical cystectomy (LRC) which is thought to lead to a faster recovery, shorter hospital stay, decreased 
morbidity, and more rapid return to daily activities, in addition to maintaining the same functional and oncological outcomes as $\operatorname{ORC}(1,2,4)$. However, despite the success numerous centres have had with LRC, it remains a matter of debate as there is no head to head trial comparing the long term outcomes and oncological results between the two modalities $(1-3,5)$.

As it stands, almost $50 \%$ of patients who undergo ORC will have a tumour recurrence, which lead to the mortality of many of these patients, while the 5-year tumour recurrence survival rates ranges between 73 and $89 \%$ in node negative organ confined disease, 45-55\% with extravesical disease, and 25-35\% with nodal involvement (14). To try to improve the survival, different centres have adopted an extended pelvic lymph node dissection (PLND) approach (1). This has also found its way in to the laparoscopic procedure; however, no long-term results are available.

The complication rate of $\mathrm{ORC}$ is in the range of $40-65 \%$ with a transfusion requirement of around $66 \%$, while the major complication rates range between $10-12 \%$ and a mortality of $2-3 \%(1,4)$. LRC can also provide an advantage of less blood loss, analgesic requirement, reduced scarring, and less complications (2,6). Despite these advantages, LRC is a technically challenging procedure that requires a high level of laparoscopic skills and has a long learning curve $(1,2)$. However, numerous reports have emerged showing success with the procedure in addition to success with urinary diversion methods $(2,5)$. Centres have attempted both intra and extracorporal urine diversion with good results $(2,5,7)$.

To this end, we report our 5-year experience of a single surgeon's LRC with PLND and urinary diversion operative results and complications and compare it to those of similar published data.

\section{MATERIALS AND METHODS}

\section{Patients}

Between February 2006 and March 2011, all patients with pathologically confirmed bladder cancer who underwent laparoscopic radical cystectomy and pelvic lymph node dissection and urinary diversion were included. The indications for radical cystectomy were patients who had muscle invasive disease, high-grade disease, or recurrent non-muscle invasive disease, i.e. CIS. Patients who refused laparoscopic cystectomy, patients with severe cardio-respiratory disease, and patients with metastatic disease were excluded. An experienced laparoscopic surgeon performed the LRC procedure. The data for all the patients were inputted prospectively into a database. All patients had a post-transurethral resection of bladder tumour (TURBT) pathologically proven and a staging computed tomography (CT) scan proven organ confined T2NOMO bladder cancer disease.

\section{Operative Procedure}

The LRC procedure is started by establishing a pneumoperitoneum and the insertion of two $5 \mathrm{~mm}$ and three $10 \mathrm{~mm}$ trocars. We use the ligadure system, harmonic scalpel, bipolar scissors, metal and plastic clips to dissect the tissue and ligation of vessels. After identifying the anatomy, we start with dissection of the seminal vesicles and posterior surface of the prostate. After dissection of the Retzius space, we incise of the pelvic fascia, dissect the prostate apex and dissect the urethra. Obturator, external, internal, common iliac, presacral, para-aortic and paracaval lymph nodes are all dissected for pathological analysis. The specimen is removed in a silicon bag. In females, the procedure is started by dissection of the uterus ligaments and peritoneum in the Douglas cavity. Bladder with the urethra, uterus, adnexa with anterior vaginal wall and lymph nodes are removed transvaginally. Urinary diversions are performed via a minilaparotomy technique with the left ureter carried on to the right side under the sigmoid colon mesentery. Ileal neobladders are formed according to the technique described by Studer et al. (8). A $14 \mathrm{~F}$ drain is left in the abdominal cavity after surgery.

\section{Outcome Measures and Analysis}

The outcome measures evaluated the patients' demographics, cystectomy pathology grading, operative time, conversion rate, blood loss, transfusion rate, urinary diversion method, morphine analgesic requirement, length of hospital stay, and complication rates. 
The complications were classified according to the Clavien classification (CC) of surgical complications (9). We considered CC I and II as minor complications and CC III and above as major.

All CT scans were reported by experienced uro-radiologists and all specimens were analysed by experienced pathologists.

\section{Follow-up}

Patients were seen in clinic one month postoperatively, then every three months for the first year, then every six months for the next year, then on a yearly basis. Follow-up investigations consisted of transabdominal ultrasound, CT and LAB Tests.

\section{Learning Curve}

To analyse the learning curve for the procedure, a comparison was conducted between the first half and the second half of the cohort. The parameters compared were the demographics, operative time, blood loss, conversion rate, length of hospital stay, and the complications. For dichotomous data, a Mantel-Haenszel Chi square was used for statistical analysis and for continuous data, an Inverse variance analysis was conducted. A P value of $<0.05$ was considered statistically significant.

\section{Review of the Literature}

A literature search for publications reporting on LRC was conducted in January 2012. The Cochrane Library, PubMed, Medline, and Scopus databases were searched. Terms used included 'laparoscopic radical cystectomy', 'laparoscopic cystectomy', 'radical cystectomy', and 'cystectomy'. Only studies reporting on 50 or more cases were included; furthermore, studies comparing LRC to other procedures were not included to allow 'likefor-like' comparison of the literature.

\section{RESULTS}

\section{Patient Data}

During the 5-year period, 60 men and 5 women were included, with a mean age of $59( \pm$ 7.89) years (Table-1). The 91\% of the patients were smokers; with an average body mass index (BMI) of 27.45, and 17\% had previous operations (Table-1).
The majority of the patients (84.6\%) had a TURBT pathology result of G2pT2, while the remaining had G3pT2 bladder cancers all of which were NOMO.

\section{Postoperative Data}

The mean operating time was 294 minutes, with four patients requiring open conversions due to technical problems due to the high BMI, in addition to no significant progress in the surgery because of clinical underestimation of the stage. Seven patients required blood transfusions and each received two units. The mean intra-operative blood loss was $249 \mathrm{~mL}$. All patients had extended lymph node dissection with an average lymph node yield of 18. The mean length of hospital stay was 9 days with an average morphine requirement of 3.7 days.

Sixty percent of the patients underwent an ileal conduit diversion, while $36.9 \%$ were submitted to neobladders , and 3.1\% to ureterocutaneostomies. All patients with neobladders remained continent with a median of 18 months (range 1-48 months) post-operative follow-up.

\section{Complications}

There were 9 intra-operative complications which were managed during the procedure and did not have any effect on the post-operative recovery (Table-2). In total, 44.6\% (29/65) of the patients developed post-operative complications. Among them, the majority were CC I (22/29), while 4 were CC II, and 3 were CC V. In total, 40\% (26/65) developed minor complications and 4.6\% (3/65) developed major complications.

\section{Pathological Data}

The pathological staging post cystectomy is on Table-1. There were no distant metastases in any of the patients. However, 14 patients had positive lymph nodes and received adjuvant chemotherapy with gemzar and cisplatinum (Table-1). All the specimens had negative margins.

\section{Nerve-sparing Data}

Only three patients underwent nerve-sparing surgery, of which two had full erections within 3 months of their surgery and the third had full erection 6 months post-operatively. However, all 3 needed the use of tadalafil initially. 
Table 1 - Patient Demographics and Operative Parameters.

Parameter

Median (range)

$\operatorname{Sex}(M: F)$

Previous Operations

$\mathrm{BMI}($ mean $\pm \mathrm{SD})$

Smokers

TURBT Path:

G2

G3

Operative Time (minutes (mean \pm SD))

Blood Loss (mL (mean \pm SD))

Extended LND

LN yield

Urinary Diversion:

Ileal Conduit

Neobladder

Ureterocutaneostomy

Length of hospital stay

Morphine Requirement (days (mean \pm ))

Cystectomy Path:

G2pT2b

G2pT3a

G2pT3a N1

G2pT3b N1

G3pT3a

G3pT2b N1

G3pT3a N1

G3pT3b N1

G3pT4a N1
$60: 5$

$16.9 \%(11 / 65)$

$27.45 \pm 2.2$

$28(23-32)$

$90.8 \%(59 / 65)$

$84.6 \%(55 / 65)$

$15.4 \%(10 / 65)$

$$
\begin{gathered}
294 \pm 27 \\
249.69 \pm 95.59
\end{gathered}
$$

65

$17.97 \pm 3.49$

39

24

2

$$
\begin{gathered}
9.42 \pm 2 \\
3.69 \pm 0.8
\end{gathered}
$$

$$
9(7-18)
$$

38

8

1

4

1

1
6

5

1

$290(240-340)$

$210(170-500)$

\section{8}

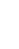

更

1

(1)


Table 2 - Complications.

\begin{tabular}{|c|c|}
\hline Intraoperative injuries: & \\
\hline Bowel & 4 \\
\hline Rectal & 3 \\
\hline Vascular & 2 \\
\hline \multicolumn{2}{|c|}{ Postoperative complications: } \\
\hline Sepsis & 3 \\
\hline Urine Retention & 1 \\
\hline Bleeding & 6 \\
\hline Thromboembolism & 4 \\
\hline Neurologic & 3 \\
\hline Muscular & 1 \\
\hline Ileus & 10 \\
\hline Cardiac & 1 \\
\hline Ureteral stenosis & 12 \\
\hline Urine Leak & 3 \\
\hline Lymph leak & 3 \\
\hline \multicolumn{2}{|c|}{ C-Classification per Patient: } \\
\hline I & 22 \\
\hline$\|$ & 4 \\
\hline IIla & 0 \\
\hline IIlb & $0(3)$ \\
\hline IVa & 0 \\
\hline IVb & 0 \\
\hline V & 3 \\
\hline
\end{tabular}

\section{Learning Curve}

Table- 3 depicts the comparison of group 1 and 2, which represent the first half and the second half of the cohort respectively. There was no difference between the two groups regarding sex, age, BMI, or previous operations. Group 2 had a sig- nificantly reduced operative time $(p=0.002)$, however no statistical significance was found regarding blood loss, conversion rates, length of hospital stay, or complication rates (Table-3).

\section{Literature Search}

Figure-1 depicts the flowchart of the article selection process. All articles excluded were due to either non-relevance to the aim of this review or reported on less than 50 LRC. Seven articles were included, their results are depicted on Table-4 (1017). No studies were found describing the learning curve for laparoscopic cystectomy procedures.

\section{DISCUSSION}

Laparoscopic surgery has advanced considerably in the last decade, extending its use even to difficult prolonged procedures such as radical cystectomies. Since the first LRC was described, numerous centres have published their centres experience with the procedure (18). However, only a handful of centres have published data on more than 50 procedures (Table-4). However, ORC remains the gold standard of treatment for select bladder cancer cases and LRC is quickly emerging as a viable and safe alternative.

We present our data on our 5 year experience with LRC all performed by one surgeon. Our data suggests that localized muscle invasive bladder cancer can be successfully treated laparoscopicly; however, there does seem to be a steep learning curve. We were able to achieve operating times, intra-operative blood loss, and length of hospital stays in par with the largest published series and better results with others. Despite this, we had a high overall complication rate of $44.6 \%$ (29/65). However, if it is take into consideration the severity of the complications, about 90\% (26/29) of the patients that developed complications were treated conservatively with no further surgical intervention required and were classified as Clavien classification I and II. The remaining three patients, representing $10 \%$ of those that developed complications and $4.6 \%$ of the overall patient cohort, required re-operations. Two of these patients were re-operated due to ileal leakage due to tight anastomosis and one due to mechanical 
Table 3 - Comparison between the initial and final cohort of patients.

\begin{tabular}{lccc}
\hline Parameter & Group 1 & Group 2 & P value \\
\hline Sex (M:F) & $36: 1$ & $34: 4$ & $P=0.21$ \\
Age & $59 \pm 8.67$ & $59 \pm 7.2$ & $P=0.99$ \\
BMI & $27.25 \pm 1.88$ & $27.64 \pm 2.49$ & $P=0.44$ \\
Previous Operations & $6 / 37$ & $5 / 38$ & $P=0.71$ \\
Operative Time (minutes (mean \pm SD)) & $303 \pm 28$ & $285 \pm 22.93$ & $P=0.002$ \\
Blood Loss (mL (mean \pm SD)) & $259.69 \pm 102.2$ & $240 \pm 89.2$ & $P=0.37$ \\
Conversions & $4 / 37$ & $0 / 38$ & $P=0.12$ \\
Length of hospital stay & $9.38 \pm 20.9$ & $9.45 \pm 1.99$ & $P=0.88$ \\
Complications & $15 / 37$ & $14 / 38$ & $P=0.74$ \\
Clavien I \& II & $13 / 37$ & $13 / 38$ & $P=0.93$ \\
Clavien > III & $2 / 37$ & $1 / 38$ & $P=0.55$ \\
\hline
\end{tabular}

obstruction. All three patients developed severe sepsis and died.

Although no statistical significance was found regarding most of the outcomes (Table-3), Figure-2 depicts the complications that occurred with a comparison to the number of procedures conducted in each year. The figure clearly shows that the number of procedures is on an upwards increasing slope, while the complications are relatively plateauing. Furthermore, it is worth mentioning that the two patients that had tight anastomosis and died were amongst the first quarter of the patient cohort operated on. This analysis shows that complications decreases as the number of patients increase.

Despite this, the major complication rate of $4.6 \%$ in our study is comparable to previous studies and remains less than that of ORC procedures (Table-4) $(1,4,17,19)$. In a comparative analysis of 50 patients in each the LRC and ORC groups, Haber et al. reported that LRC had a minor complication rate of $18 \%$ while ORC had 22\%. Furthermore, they reported that LRC had a major complication rate of $8 \%$ compared to $6 \%$ of that of ORC. However, neither comparison reached statistical significance.

Huang et al. reported the largest series to date, where 171 patients underwent LRC. Their median operative time was 325 minutes with a median blood loss of $270 \mathrm{~mL}$. Their study was the only other study to classify their complications into the Clavien classification. They reported a total complication rate of 39.2\% (67/171), with 6.4\% (11/171) CC I complications, 19.3\% (33/171) CC II complications, and 13.5\% (23/171) CC III complications.

Interestingly, these results matched the same author's initial results of 85 patients, where their overall complication rate was $14.1 \%$ as opposed to their larger report with $39.2 \%$ complication rate, with similar operative times, blood loos, and post-operative hospital stay.

Castello et al. data from their initial report and their follow-up report with 59 and 85 patients respectively showed that there seems to be an improvement in their operative time and a decrease in the complication rates (Table-1). However, all 
Figure 1 - Flowchart for literature review.

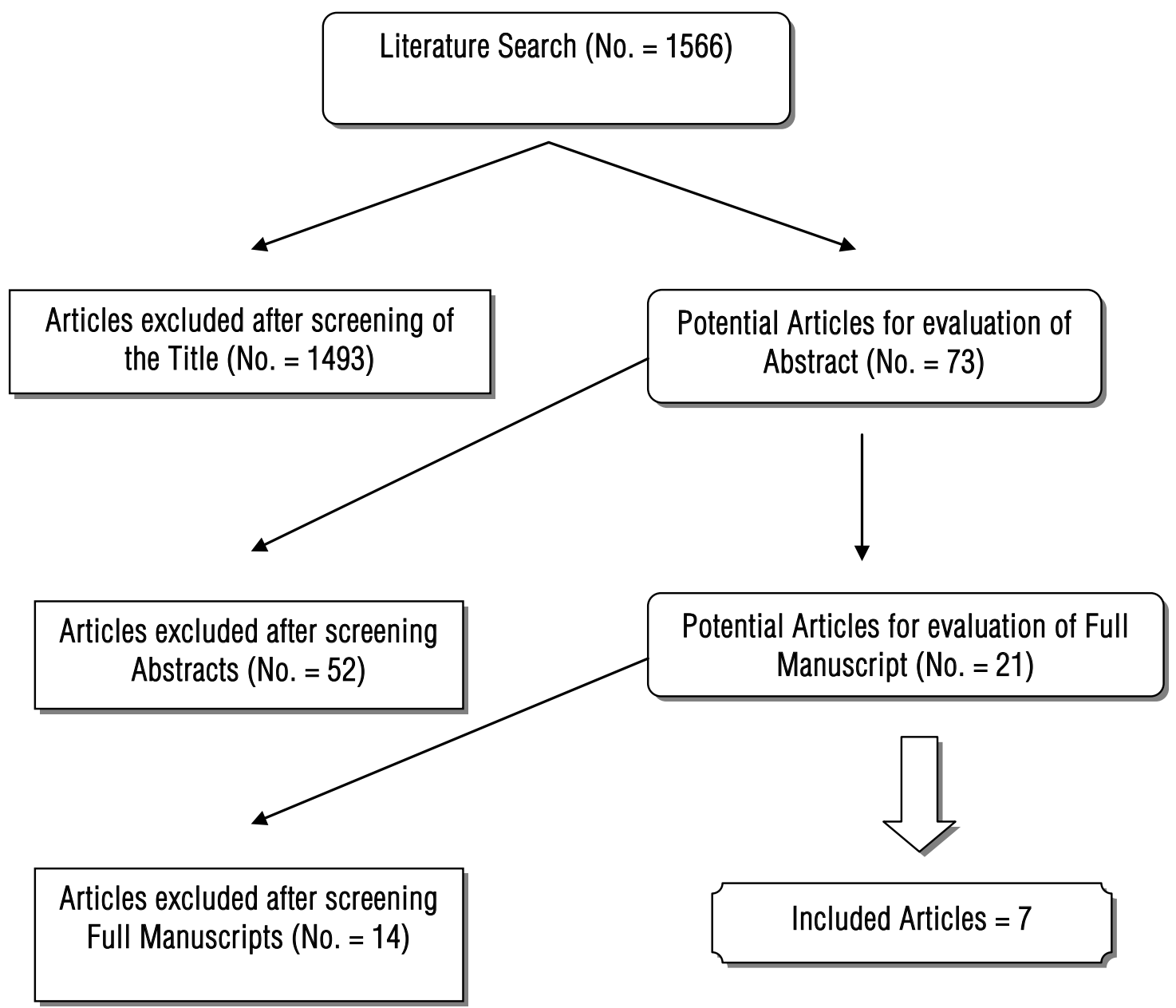

studies reported more complications in patients who had neobladders, as compared to those with ileal conduits. This abode the same for our cohort, as more complications were noted in patients who were submitted to neobladders as opposed to those with conduits. This would explain why Huang et al. have a higher complication rate than other studies, as they only performed neobladder procedures.

It is evident that there is a variation between the studies regarding operative times and blood loss, as well as complication rates. This not only portrays the varying expertise of the surgeons and centres, but also emphasizes that there is no standardize operative procedure and techniques; furthermore, there is no consensus on which urinary diversion technique is best suited for laparoscopic surgery.

Though Castello et al. started their study in 1997 and published their work up until 2005, this period remains short with inadequate data to be able to compare between the oncological results of LRC to those of the open counterpart. A systematic review conducted by Chade et al., which included 19 studies comparing ORC to LRC as well as robotic 


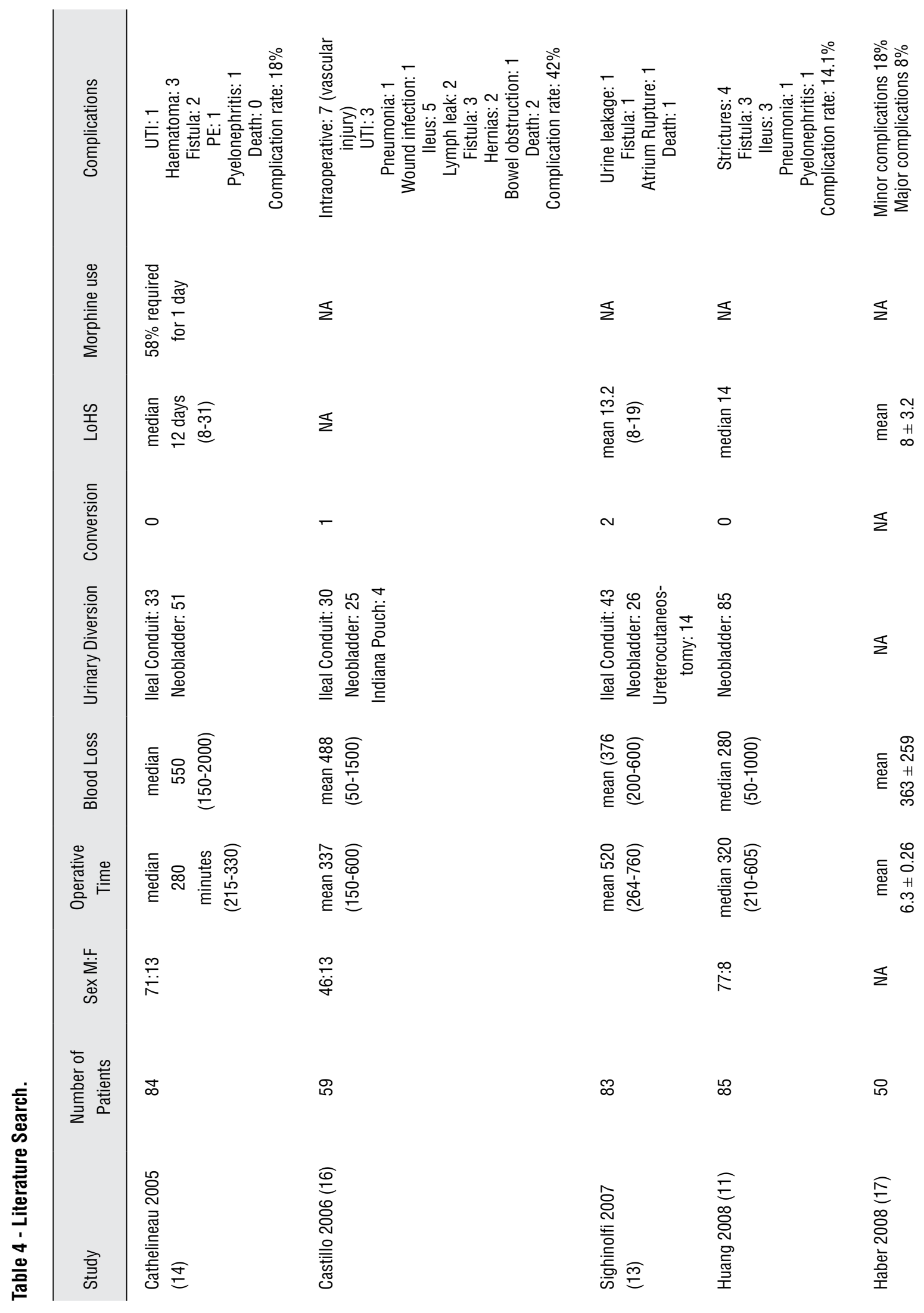




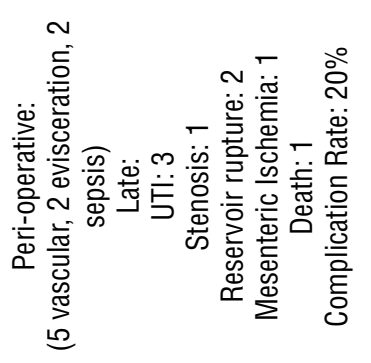
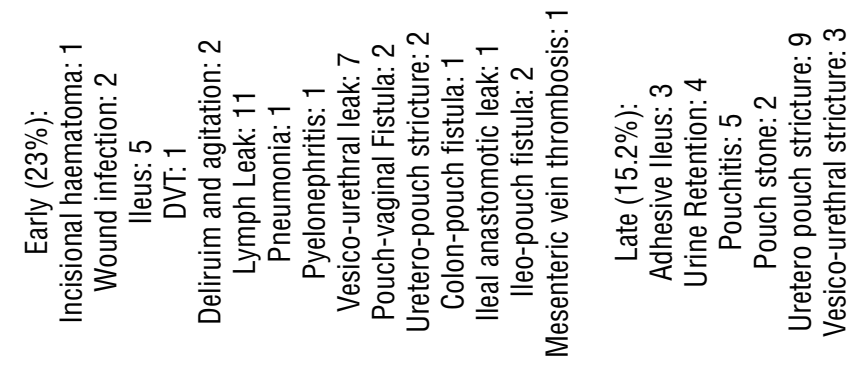

$\circ$

$\Sigma$

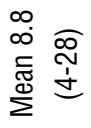

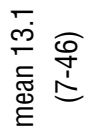

$\circ$

$\circ$

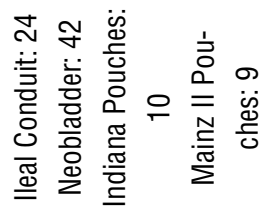

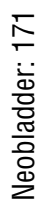

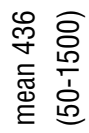

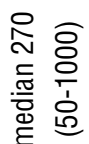

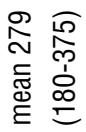

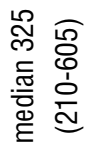

옴

$\frac{}{\stackrel{0}{n}}$

$\infty$

즌

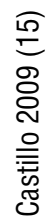

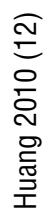

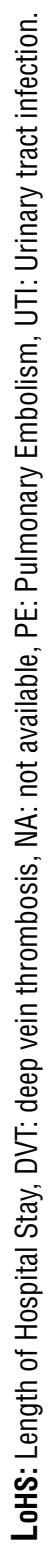


Figure 2 - Yearly depiction of number of procedures.

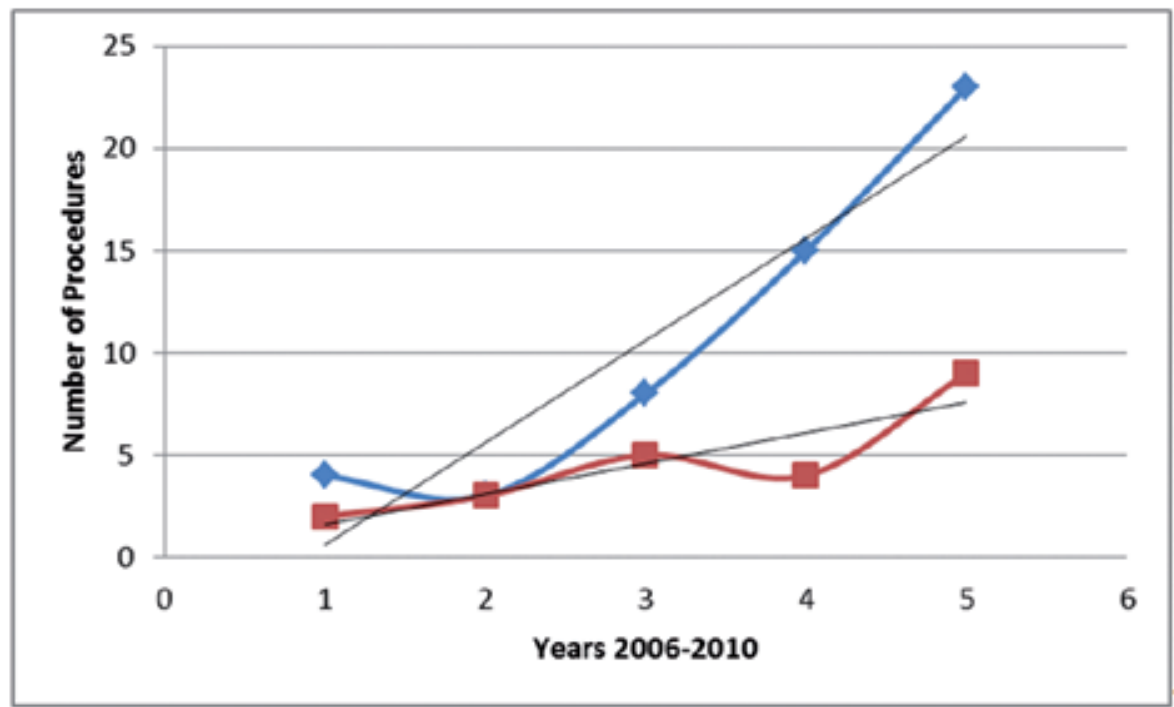

radical cystectomy (RRC), was not able to show superiority of one modality to the other (3). Furthermore, they stipulate that despite the select cohort of patients that were included in the LRC and RRC groups compared to the non-selective ORC group, they were unable to see a clear impact regarding oncological outcomes. However, they do mention that the LRC and RRC results are encouraging and that continual follow-up of these patients will allow an improved statistical analysis. Haber et al. reported that though LRC was significantly longer than ORC, it had significantly less blood loss and transfusion requirement, however no other difference was found between the two procedures (17).

Bladder cancer management has a narrow opportunity for effective cancer control, as the outcomes are highly dependent on the radical cystectomy, in addition to a lack of salvage procedures for recurrence, which emphasizes on the need for more adequate precise resection during the initial operation (3). What is evident is that with time and improved skills and experience of surgeons, LRC might replace ORC. With the emergence of robotics, this procedure is rapidly replacing laparoscopy due to its shorter learning curve and at least similar if not better operative and post-operative results. In a comparative analysis between LRC and RRC, Abraham et al. found that both procedures could be performed safely without compromising the oncological results. Furthermore, they found that RRC has a shorter learning curve, less blood loss, complications, and earlier return of bowel function (20). Furthermore, the only randomised trial found in the literature comparing robotic radical cystectomy to open radical cystectomy showed that RRC had significantly improved outcomes regarding blood loss, operative time, analgesic use, with no difference regarding complications, length of hospital stay or pathological outcome (21). Despite these studies, a larger multi-institutional analysis comparing laparoscopic, open, and robotic radical cystectomy is needed to establish superiority of one procedure over the others.

Therefore, time will tell how far minimally invasive procedures will progress; until then, ORC still remains gold standard, and in experienced hands can have a low blood loss as well as a short hospital stay in par with LRC or RRC. 


\section{CONCLUSION}

LRC is safe and efficient modality of treatment of bladder cancer that can be add to the urologist's arsenal of treatment options. Though there comes with it, a steep learning curve, once overcome, can provide an alternative to ORC. LRC though comes with a prolonged operative time, can also benefit from minimal blood loss as well as a complication rate similar to ORC if not better. However, longer follow-ups and larger cohort studies are needed to further evaluate its precise benefits over ORC and LRC is finding its way into many centres and time will tell of its full potential.

\section{CONFLICT OF INTEREST}

\section{None declared.}

\section{REFERENCES}

1. Challacombe BJ, Bochner BH, Dasgupta P, Gill I, Guru K, Herr $H$, et al.: The role of laparoscopic and robotic cystectomy in the management of muscle-invasive bladder cancer with special emphasis on cancer control and complications. Eur Urol. 2011; 60: 767-75.

2. Hemal AK: Robotic and laparoscopic radical cystectomy in the management of bladder cancer. Curr Urol Rep. 2009; 10: 45-54

3. Chade DC, Laudone VP, Bochner BH, Parra RO: Oncological outcomes after radical cystectomy for bladder cancer: open versus minimally invasive approaches. J Urol. 2010; 183: 862-69.

4. Irwin BH, Gill IS, Haber GP, Campbell SC: Laparoscopic radical cystectomy: current status, outcomes, and patient selection. Curr Treat Options Oncol. 2009; 10: 243-55.

5. Berger A, Aron M: Laparoscopic radical cystectomy: longterm outcomes. Curr Opin Urol. 2008; 18: 167-72.

6. Puppo P, Introini C, Naselli A: Surgery insight: advantages and disadvantages of laparoscopic radical cystectomy to treat invasive bladder cancer. Nat Clin Pract Urol. 2007; 4: 387-94.

7. Ríos González E, López-Tello García JJ, Martínez-Piñeiro Lorenzo L: Laparoscopic radical cystectomy. Clin Transl Oncol. 2009; 11: 799-804.

8. Studer UE, Ackermann D, Casanova GA, Zingg EJ: Three years' experience with an ileal low pressure bladder substitute. $\mathrm{Br} \mathrm{J}$ Urol. 1989; 63: 43-52.

9. Dindo D, Demartines N, Clavien PA: Classification of surgical complications: a new proposal with evaluation in a cohort of 6336 patients and results of a survey. Ann Surg. 2004; 240: 205-13.
10. Cathelineau X, Jaffe J: Laparoscopic radical cystectomy with urinary diversion: what is the optimal technique? Curr Opin Urol. 2007; 17: 93-7.

11. Huang J, Lin T, Xu K, Huang H, Jiang C, Han J, et al.: Laparoscopic radical cystectomy with orthotopic ileal neobladder: a report of 85 cases. J Endourol. 2008; 22: 939-46.

12. Huang J, Lin T, Liu H, Xu K, Zhang C, Jiang C, et al.: Laparoscopic radical cystectomy with orthotopic ileal neobladder for bladder cancer: oncologic results of 171 cases with a median 3-year follow-up. Eur Urol. 2010; 58: 442-9.

13. Sighinolfi MC, Micali S, Celia A, DeStefani S, Grande M, Rivalta $M$, et al.: Laparoscopic radical cystectomy: an Italian survey. Surg Endosc. 2007; 21: 1308-11.

14. Cathelineau X, Arroyo C, Rozet F, Barret E, Vallancien G: Laparoscopic assisted radical cystectomy: the montsouris experience after 84 cases. Eur Urol. 2005; 47: 780-4.

15. Castillo OA, Vitagliano G, Vidal-Mora I: Laparoscopic radical cystectomy. The new gold standard for bladder carcinoma? Arch Esp Urol. 2009; 62: 737-44.

16. Castillo OA, Abreu SC, Mariano MB, Tefilli MV, Hoyos J, Pinto I, et al.: Complications in laparoscopic radical cystectomy. The South American experience with 59 cases. Int Braz J Urol. 2006; 32: 300-5

17. Haber GP, Crouzet S, Gill IS: Laparoscopic and robotic assisted radical cystectomy for bladder cancer: a critical analysis. Eur Urol. 2008; 54: 54-62.

18. Puppo P, Naselli A: Laparoscopic radical cystectomy. Where do we stand? Arch Esp Urol. 2010; 63: 508-19.

19. Fergany AF: Laparoscopic radical cystectomy. Arab Journal of Urology, 2012; 1: 40-5.

20. Abraham JB, Young JL, Box GN, Lee HJ, Deane LA, Ornstein DK: Comparative analysis of laparoscopic and robot-assisted radical cystectomy with ileal conduit urinary diversion. J Endourol. 2007; 21: 1473-80.

21. Nix J, Smith A, Kurpad R, Nielsen ME, Wallen EM, Pruthi RS: Prospective randomized controlled trial of robotic versus open radical cystectomy for bladder cancer: perioperative and pathologic results. Eur Urol. 2010; 57: 196-201.

\footnotetext{
Correspondence address

Dr. Omar M. Aboumarzouk Urology Department, Royal Bournemouth Hospital, Castle Lane East, Bournemouth, BH7 7DW, UK Urology Department, Wales Deanery, Cardiff, Wales, UK Fax: +44 29 2034-3430 E-mail: aboumarzouk@gmail.com
} 